\title{
An Improved Procedure for the Three-Component Synthesis of Highly Substituted Pyridines Using Ionic Liquid
}

\author{
Brindaban C. Ranu, ${ }^{*}$ Ranjan Jana and S. Sowmiah
}

Supporting Information

\section{Table of Contents}

Spectroscopic (IR, ${ }^{1} \mathrm{H}$ NMR, ${ }^{13} \mathrm{C}$ NMR and HRMS) Data

Page no.

of All Products in Table 1

S2-S6

${ }^{13}$ C NMR Spectra of All Products in Table 1

S7-S16 
IR, ${ }^{1} \mathrm{H},{ }^{13} \mathrm{C}$ and melting points of all compounds and HRMS data of unknown compounds in table 1 are given below according to their entries

2-Amino-4-phenyl-6-phenylsulfanyl-pyridine-3,5-dicarbonitrile (Entry 1, Table 1):

Colourless solid, mp $\left(\mathrm{CH}_{3} \mathrm{CN}\right)$ 216-218 ${ }^{\circ} \mathrm{C}$; IR (KBr): 3479, 3350, 3213, 2218, 1622, 1579, 1548, 1521, 1263, 758, $692 \mathrm{~cm}^{-1}$; ${ }^{1} \mathrm{H}$ NMR (300 MHz, DMSO-d 6 ): $\delta 7.27$ (broad, 2H), 7.38-7.47 (m, 10H); ${ }^{13} \mathrm{C}$ NMR (75 MHz): $\delta$ 87.3, 94.0, $115.3(2 \mathrm{C}), 127.5,128.5$ (2C), 128.9 (2C), 129.4, 129.7, 130.6 (2C), 133.9, 135.3 (2C), 158.4, 160.0, 167.4.

2-Amino-6-(4-chloro-phenylsulfanyl)-4-phenyl-pyridine-3,5-dicarbonitrile (Entry 2, Table1): White solid, $\mathrm{mp}\left(\mathrm{CH}_{3} \mathrm{CN}\right) 228-230{ }^{\circ} \mathrm{C}$; IR (KBr): 3481, 3352, 3214, 2218, 1621, 1570, 1548, 1262, 756, $693 \mathrm{~cm}^{-1}$; ${ }^{1} \mathrm{H}$ NMR (300 MHz, DMSO-d 6 ): $\delta 6.34$ (broad, 2H), 7.21-7.40 (m, 9H); ${ }^{13} \mathrm{C}$ NMR (75 MHz): $\delta$ 87.0, 94.3, 114.5, 114.8, 125.5, 128.0 (2C), 128.4 (2C), 128.9 (2C), 130.3, 133.0, 135.6, 136.5 (2C), 158.0, 159.3, 167.2.

2-Amino-6-benzylsulfanyl-4-phenyl-pyridine-3,5-dicarbonitrile (Entry 3, Table 1): Colourless solid, mp $\left(\mathrm{CH}_{3} \mathrm{CN}\right)$ 193-195 ${ }^{\circ} \mathrm{C}$; IR (KBr): 3450, 3334, 3211, 2956, 2191, 1633, 1348, $734 \mathrm{~cm}^{-1} ;{ }^{1} \mathrm{H}$ NMR (300 MHz, DMSO-d 6 ): $\delta 3.77$ (s, 2H), 7.33-7.42 (m, 10H), 7.57 (broad, 2H); ${ }^{13} \mathrm{C}$ NMR (75 MHz): $\delta 42.2,86.4,93.7,114.4,115.7,127.0$, 128.6 (2C), 128.8 (2C), 129.0, 129.3 (2C), 130.7 (2C), 131.7, 134.3, 137.7, 160.0, 166.7.

2-Amino-6-(2-amino-phenylsulfanyl)-4-phenyl-pyridine-3,5-dicarbonitrile (Entry 4, Table 1): Colourless solid, mp $\left(\mathrm{CH}_{3} \mathrm{CN}\right) 224-225{ }^{\circ} \mathrm{C}$; IR (KBr): 3392, 3327, 3220, 2923 , 2212, 1637, 1542, 1523, 754, $704 \mathrm{~cm}^{-1}$; ${ }^{1} \mathrm{H}$ NMR (300 MHz, DMSO-d 6 ): $\delta 5.36$ (broad, 2H), $6.55(\mathrm{t}, J=6.9 \mathrm{~Hz}, 1 \mathrm{H}), 6.77(\mathrm{~d}, J=7.7 \mathrm{~Hz}, 1 \mathrm{H}), 7.08-7.33(\mathrm{~m}, 5 \mathrm{H}), 7.50-7.53(\mathrm{~m}$, 2H), 7.62 (broad, 2H), ${ }^{13} \mathrm{C}$ NMR (75 MHz): $\delta$ 86.7, 93.9, 107.9, 116.7, 116.2, 117.7, 126.2, 128.2 (2C), 128.7 (2C), 129.0, 129.9, 134.4, 137.5, 151.5, 158.7, 159.9, 167.1.

2-Amino-4-(4-chloro-phenyl)-6-phenylsulfanyl-pyridine-3,5-dicarbonitrile (Entry 5, Table 1): Colourless solid, mp $\left(\mathrm{CH}_{3} \mathrm{CN}\right) 222-224{ }^{\circ} \mathrm{C}$; IR (KBr): 3485, 3344, 3220, 2925 , 
2214, 1633, 1544, 1494, 1257, $1093 \mathrm{~cm}^{-1} ;{ }^{1} \mathrm{H}$ NMR (300 MHz, DMSO-d 6 ): $\delta$ 7.48-7.55 (m, 3H), 7.57-7.64 (m, 6H), 7.79 (broad, 2H); ${ }^{13} \mathrm{C}$ NMR (75 MHz): $\delta$ 87.4, 93.6, 115.2, 115.5, 127.3, 129.2 (2C), 129.5 (2C), 129.8, 130.8 (2C), 133.1, 135.2 (2C), 135.7, 157.8, 159.9, 166.6.

2-Amino-4-(4-nitro-phenyl)-6-phenylsulfanyl-pyridine-3,5-dicarbonitrile (Entry 6, Table 1): Colourless solid, $\mathrm{mp}\left(\mathrm{CH}_{3} \mathrm{CN}\right) 287-289^{\circ} \mathrm{C}$; $\mathrm{IR}(\mathrm{KBr}): 3406,3328,3234,2227$, 2214, 1645, 1552, 1350, $756 \mathrm{~cm}^{-1} ;{ }^{1} \mathrm{H}$ NMR (300 MHz, DMSO-d 6 ): $\delta$ 7.46-7.48 (m, 3H), 7.56-7.59 (m, 2H), 7.85 (d, $J=8.6 \mathrm{~Hz}, 2 \mathrm{H}), 7.90$ (broad, 2H), 8.39 (d, $J=8.6 \mathrm{~Hz}, 2 \mathrm{H}$ );

${ }^{13} \mathrm{C}$ NMR (75 MHz): $\delta$ 87.2, 93.3, 114.9, 115.3, 124.2 (2C), 127.2, 129.8 (2C), 130.1, 130.5 (2C), 135.1 (2C), 140.5, 148.9, 157.0, 159.8, 166.5 .

\section{2-Amino-4-(4-methylsulfanyl-phenyl)-6-phenylsulfanyl-pyridine-3,5-dicarbonitrile}

(Entry 7, Table 1): Colourless solid; $\mathrm{mp}\left(\mathrm{CH}_{3} \mathrm{CN}\right) 254-256{ }^{\circ} \mathrm{C}$; $\mathrm{IR}(\mathrm{KBr}): 3408$, 3332, 3247, 3151, 2218, 2215, 1651, 1573, 1552, 1481, $1294 \mathrm{~cm}^{-1}$; ${ }^{1} \mathrm{H}$ NMR $(300 \mathrm{MHz}$, DMSO-d $\left._{6}\right): \delta 2.55(\mathrm{~s}, 3 \mathrm{H}), 7.43(\mathrm{~d}, J=8.3 \mathrm{~Hz}, 2 \mathrm{H}), 7.48-7.50(\mathrm{~m}, 4 \mathrm{H}), 7.58-7.61(\mathrm{~m}$, $3 \mathrm{H}), 7.78$ (broad, 2H); ${ }^{13} \mathrm{C}$ NMR (75 MHz): $\delta 14.4,87.4,93.7,115.5,115.8,125.6(2 \mathrm{C}$ ), 127.6, 129.4 (2C), 129.8 (2C), 130.1, 130.2, 135.2 (2C), 142.2, 158.5, 160.1, 166.5. HRMS Calcd for $\mathrm{C}_{20} \mathrm{H}_{14} \mathrm{~N}_{4} \mathrm{~S}_{2}$ : [M+H] $]^{+}$, 375.0738; Found: 375.0728.

2-Amino-4-(4-hydroxy-phenyl)-6-phenylsulfanyl-pyridine-3,5-dicarbonitrile (Entry 8, Table 2): Colourless solid, $\mathrm{mp}\left(\mathrm{CH}_{3} \mathrm{CN}\right) 315-316{ }^{\circ} \mathrm{C}$; $\mathrm{IR}$ (KBr): 3648, 3496, 3367 , 3236, 2222, 2216, 1631, 1609, 1552, $758 \mathrm{~cm}^{-1}$; ${ }^{1} \mathrm{H}$ NMR (300 MHz, DMSO-d 6 ): $\delta 6.93$ (d, $J=8.5 \mathrm{~Hz}, 2 \mathrm{H}), 7.39(\mathrm{~d}, J=8.5 \mathrm{~Hz}, 2 \mathrm{H}), 7.50(\mathrm{~m}, 3 \mathrm{H}), 7.60(\mathrm{~m}, 2 \mathrm{H}), 7.69$ (broad, $2 \mathrm{H}), 10.04$ (bs, $1 \mathrm{H}) ;{ }^{13} \mathrm{C}$ NMR (75 MHz): $\delta$ 93.7, 96.9, 115.5 (2C), 115.8, 116.0, 124.5, 127.6, 129.8 (2C), 130.0, 130.7 (2C), 135.2 (2C), 159.0, 159.8, 160.2, 166.5.

\section{2-Amino-4-benzo[1,3] dioxol-5-yl-6-phenylsulfanyl-pyridine-3,5-dicarbonitrile}

(Entry 9, Table 1): Colourless solid, $\mathrm{mp}\left(\mathrm{CH}_{3} \mathrm{CN}\right) 234-236{ }^{\circ} \mathrm{C}$; $\mathrm{IR}(\mathrm{KBr}): 3479,3350$, $3213,2218,1622,1548,1263,758,692,451 \mathrm{~cm}^{-1} ;{ }^{1} \mathrm{H}$ NMR (300 MHz, DMSO-d 6 ): $\delta$ $6.14(\mathrm{~s}, 2 \mathrm{H}), 7.01-7.16(\mathrm{~m}, 3 \mathrm{H}), 7.47-7.49(\mathrm{~m}, 3 \mathrm{H}), 7.57-7.60(\mathrm{~m}, 2 \mathrm{H}), 7.74$ (broad, $2 \mathrm{H})$; 
${ }^{13} \mathrm{C}$ NMR (75 MHz): $\delta 88.0,94.4,102.6,109.4,109.7,116.0,116.2,123.9,128.0,128.1$, 130.3 (2C), 130.5, 135.6 (2C), 148.2, 149.8, 159.0, 160.5, 166.9.

2-Amino-4-(4-methoxy-phenyl)-6-phenylsulfanyl-pyridine-3,5-dicarbonitrile (Entry 10, Table 1): Colourless solid, mp $\left(\mathrm{CH}_{3} \mathrm{CN}\right) 238-240{ }^{\circ} \mathrm{C}$; IR (KBr): 3440, 3330, 3226, 2227, 2214, 1641, 1548, 1512, 1259, 1190, 1018, $756 \mathrm{~cm}^{-1}$; ${ }^{1} \mathrm{H}$ NMR (300 MHz, DMSO$\left.\mathrm{d}_{6}\right): \delta 3.82(\mathrm{~s}, 3 \mathrm{H}), 7.94(\mathrm{~d}, J=8.4 \mathrm{~Hz}, 2 \mathrm{H}), 7.47-7.56(\mathrm{~m}, 7 \mathrm{H}), 7.68(\mathrm{broad}, 2 \mathrm{H}) ;{ }^{13} \mathrm{C}$ NMR (75 MHz): $\delta 55.5,87.1,93.5,114.2$ (2C), 115.7, 125.9, 127.3, 129.6 (2C), 129.8, 130.4 (2C), 134.9 (2C), 158.5, 159.9, 161.0, 166.3.

2-Amino-6-benzylsulfanyl-4-(4-methoxy-phenyl)-pyridine-3,5-dicarbonitrile (Entry 11, Table 1): Colourless solid, mp $\left(\mathrm{CH}_{3} \mathrm{CN}\right) 228-230{ }^{\circ} \mathrm{C}$; IR (KBr): 3450, 3334, 3211, 2956, 2191, 1633, $734 \mathrm{~cm}^{-1} ;{ }^{1} \mathrm{H}$ NMR (300 MHz, DMSO-d $)$ ): $\delta 3.65$ (s, 3H), 4.26 (s, 2H), 6.55 (broad, 2H), $6.81(\mathrm{~d}, J=8.7 \mathrm{~Hz}, 2 \mathrm{H}), 7.07-7.13(\mathrm{~m}, 3 \mathrm{H}), 7.19-7.24(\mathrm{~m}, 2 \mathrm{H}), 7.25(\mathrm{~d}$, $J=8.7 \mathrm{~Hz}, 2 \mathrm{H}) ;{ }^{13} \mathrm{C} \mathrm{NMR}(75 \mathrm{MHz}): \delta 33.9,54.8,85.7,94.4,113.7$ (2C), 114.9, 115.3, 125.0, 126.9, 128.0 (2C), 128.6 (2C), 129.6 (2C), 136.1, 157.3, 159.3, 160.8, 167.3.

HRMS Calcd for $\mathrm{C}_{21} \mathrm{H}_{16} \mathrm{~N}_{4} \mathrm{OS}:[\mathrm{M}+\mathrm{H}]^{+}, 373.1123$; Found: 373.1108 .

2-Amino-6-phenylsulfanyl-4-thiophen-2-yl-pyridine-3,5-dicarbonitrile (Entry 12,

Table 1): Colourless solid, mp $\left(\mathrm{CH}_{3} \mathrm{CN}\right) 208-210{ }^{\circ} \mathrm{C}$; IR (KBr): 3408, 3332, 3247, 3151, 2218, 2215, 1651, 1573, 1552, 1481, $1294 \mathrm{~cm}^{-1}$; ${ }^{1} \mathrm{H}$ NMR (300 MHz, DMSO-d 6 ): $\delta 6.40$ (broad, 2H), $6.97(\mathrm{t}, J=4.5 \mathrm{~Hz}, 1 \mathrm{H}), 7.20-7.22(\mathrm{~m}, 3 \mathrm{H}), 7.29-7.32(\mathrm{~m}, 3 \mathrm{H}), 7.43(\mathrm{~d}, J=$ $4.9 \mathrm{~Hz}, 1 \mathrm{H}) ;{ }^{13} \mathrm{C}$ NMR $(75 \mathrm{MHz}): \delta 86.1,93.5,114.7,114.9,126.7,127.2,128.6(2 \mathrm{C})$, 129.1, 129.4, 130.4, 132.2, 134.8 (2C), 149.8, 159.3, 167.9.

HRMS Calcd for $\mathrm{C}_{17} \mathrm{H}_{10} \mathrm{~N}_{4} \mathrm{~S}_{2}$ : [M+H] $]^{+}$, 335.0425; Found: 335.0446 .

\section{2-Amino-4-(4-hydroxy-3-methoxy-phenyl)-6-phenylsulfanyl-pyridine-3,5-}

dicarbonitrile (Entry 13, Table 1): Colourless solid, mp $\left(\mathrm{CH}_{3} \mathrm{CN}\right)$ 218-220 ${ }^{\circ} \mathrm{C}$; IR (KBr): 3648, 3496, 3367, 3236, 2222, 2216, 1631, 1609, 1552, 1354, 1266, 1109, 758 $\mathrm{cm}^{-1} ;{ }^{1} \mathrm{H}$ NMR (300 MHz, DMSO-d 6 ): $\delta 3.88$ (s, 3H), 6.64-6.78 (m, 1H), 6.90-7.01 (m, 2H), 7.17 (s, 1H), 7.33-7.43 (m, 2H), $7.58(\mathrm{t}, J=3.5 \mathrm{~Hz}, 2 \mathrm{H}), 7.69$ (broad, 2H), 9.69 
(broad, $1 \mathrm{H}) ;{ }^{13} \mathrm{C}$ NMR $(75 \mathrm{MHz}): \delta 56.6,87.7,94.2,113.8,116.3,116.5,122.7,125.2$, 128.1, 130.2 (2C), 130.3, 131.0, 135.6 (2C), 148.2, 149.6, 159.4, 160.7, 166.9. HRMS Calcd for $\mathrm{C}_{20} \mathrm{H}_{15} \mathrm{~N}_{4} \mathrm{O}_{2} \mathrm{~S}$ : [M+H] ${ }^{+}, 375.0916$; Found: 375.0909 .

2-Amino-4-(3-bromo-phenyl)-6-phenylsulfanyl-pyridine-3,5-dicarbonitrile (Entry 14, Table 1): Colourless solid, $\mathrm{mp}\left(\mathrm{CH}_{3} \mathrm{CN}\right) 256-258{ }^{\circ} \mathrm{C}$; $\mathrm{IR}(\mathrm{KBr}): 3360,3058,2212$, 2208, 1620, 1544, 1516, 1264, 760, $704 \mathrm{~cm}^{-1} ;{ }^{1} \mathrm{H}$ NMR (300 MHz, DMSO-d 6 ): $\delta 7.46-$ 7.48 (m, 6H), 7.56 (broad, 2H), 7.67 (s, 1H), 7.70-7.72 (m, 2H); ${ }^{13} \mathrm{C}$ NMR (75 MHz): $\delta$ 85.5, 92.0, 113.3, 113.4, 120.6, 125.8, 127.1 (2C), 128.0, 129.1, 129.5, 131.7, 131.9, 133.5 (2C), 134.3, 158.2, 159.8, 166.4 .

HRMS Calcd for $\mathrm{C}_{19} \mathrm{H}_{11} \mathrm{BrN}_{4} \mathrm{~S}$ : [M+H] $]^{+}$, 406.9966; Found: 406.9968 .

2-Amino-4-(2-bromo-phenyl)-6-phenylsulfanyl-pyridine-3,5-dicarbonitrile (Entry 15, Table 1): Colourless solid, mp $\left(\mathrm{CH}_{3} \mathrm{CN}\right) 242-244{ }^{\circ} \mathrm{C}$; $\mathrm{IR}(\mathrm{KBr}): 3440,3336,3217$, 2214, 1627, 1596, 1542, 1263, $758 \mathrm{~cm}^{-1} ;{ }^{1} \mathrm{H}$ NMR (300 MHz, DMSO-d 6 ): $\delta$ 7.43-7.60 (m, 8H), $7.80(\mathrm{~d}, J=7.8 \mathrm{~Hz}, 1 \mathrm{H}), 7.91$ (broad, $2 \mathrm{H}) ;{ }^{13} \mathrm{C} \mathrm{NMR}(75 \mathrm{MHz}): \delta 88.2,94.2$, 114.6, 114.9, 121.1, 127.2, 128.8, 129.9 (2C), 130.2, 130.5, 132.4, 133.3, 135.3 (2C), $135.5,158.4,159.8,166.4$.

HRMS Calcd for $\mathrm{C}_{19} \mathrm{H}_{11} \mathrm{BrN}_{4} \mathrm{~S}$ : [M+H] $]^{+}$, 406.9966; Found: 406.9961.

2-Amino-6-phenylsulfanyl-4-p-tolyl-pyridine-3,5-dicarbonitrile (Entry 16, Table 1): Colourless solid, mp $\left(\mathrm{CH}_{3} \mathrm{CN}\right) 208-21{ }^{\circ} \mathrm{C}$; IR (KBr): 3441, 3337, 3217, 2214, 1627, 1542, 1521, 1263, 1095, $758 \mathrm{~cm}^{-1} ;{ }^{1} \mathrm{H}$ NMR (300 MHz, DMSO-d $\left.\mathrm{d}_{6}\right): \delta 2.52$ (s, 3H), 7.327.74 (m, 6H), 7.45-7.55 (m, 3H), 7.63 (broad, 2H); ${ }^{13} \mathrm{C}$ NMR (75 MHz): $\delta$ 14.9, 87.6, 94.1, 115.9, 116.0, 126.0 (2C), 128.1, 129.7 (2C), 130.1 (2C), 130.3, 130.5, 135.6 (2C), $142.7,158.6,160.6(2 \mathrm{C})$.

2-Amino-6-(4-chloro-phenylsulfanyl)-4-(2-methoxy-naphthalen-1-yl)-pyridine-3,5dicarbonitrile (Entry 17, Table 1): Colourless solid, $\mathrm{mp}\left(\mathrm{CH}_{3} \mathrm{CN}\right) \quad 257-260{ }^{\circ} \mathrm{C}$; IR (KBr): 3440, 3328, 3217, 2221, 2206, 1627, 1544, 1521, 1494, 1263, $696 \mathrm{~cm}^{-1}$; ${ }^{1} \mathrm{H}$ NMR (300 MHz, DMSO-d $\left.{ }_{6}\right): \delta 3.92(\mathrm{~s}, 3 \mathrm{H}), 7.36(\mathrm{~d}, J=8.1 \mathrm{~Hz}, 1 \mathrm{H}), 7.42-7.62(\mathrm{~m}, 5 \mathrm{H}), 7.62-$ 
$7.69(\mathrm{~m}, 2 \mathrm{H}), 7.81($ broad, $2 \mathrm{H}), 7.99(\mathrm{~d}, J=7.8 \mathrm{~Hz}, 1 \mathrm{H}), 8.20(\mathrm{~d}, J=7.8 \mathrm{~Hz}, 1 \mathrm{H}) ;{ }^{13} \mathrm{C}$ NMR (75 MHz): $\delta$ 56.1, 88.7, 94.4, 113.1, 114.0, 114.2, 122.4, 123.6, 125.4, 127.4, $127.7,127.9,128.0$ (2C), 130.3, 131.7, 134.3, 136.0, 136.2 (2C), 153.1, 155.1, 159.2, 165.0 .

HRMS Calcd for $\mathrm{C}_{20} \mathrm{H}_{14} \mathrm{~N}_{4} \mathrm{~S}$ : [M+Na] $]^{+}$, 465.0553; Found: 464.6729 .

\section{2-Amino-4-(2,6-dichloro-phenyl)-6-phenylsulfanyl-1,4-dihydro-pyridine-3,5-}

dicarbonitrile (Entry 18, Table 1): Colourless solid, mp $\left(\mathrm{CH}_{3} \mathrm{CN}\right) 319-320{ }^{\circ} \mathrm{C}$; IR (KBr): 3460, 3359, 2208, 2171, 1647, 1490, 1438, 1249, $779 \mathrm{~cm}^{-1}$; ${ }^{1} \mathrm{H}$ NMR (300 MHz, DMSO-d ${ }_{6}$ ): $\delta 5.64$ (s, 1H), 5.99 (broad, 2H), 7.37 (q, $J=7.0 \mathrm{~Hz}, 7.49-7.60$ (m, 8H), 9.21 (bs, $1 \mathrm{H}) ;{ }^{13} \mathrm{C}$ NMR (75 MHz): $\delta 39.7,53.0,88.8,118.3,120.7,129.5,130.5,130.7$ (2C), $131.1,131.5$ (2C), 135.9, 136.0 (2C), 143.6 (2C), 152.3 (2C).

\section{2-Amino-6-(4-chloro-phenylsulfanyl)-4-(2,6-dichloro-phenyl)-1,4-dihydro-pyridine-} 3,5-dicarbonitrile (Entry 19, Table 1): Colourless solid, $\mathrm{mp}\left(\mathrm{CH}_{3} \mathrm{CN}\right) 334-335{ }^{\circ} \mathrm{C}$; IR (KBr): 3448, 3361, 2208, 2165, 1647, 1488, 1481, 1436, 1251, 1093, 819, 781; ${ }^{1} \mathrm{H}$ NMR (300 MHz, DMSO-d ${ }_{6}$ ): $\delta 5.61$ (s, 1H), 5.98 (broad, 2H), 7.33-7.38 (m, 2H), 7.48-7.53 (m, 4H), 9.19 (broad, 2H); ${ }^{13} \mathrm{C}$ NMR (75 MHz): $\delta 38.4,51.7,87.5,116.8,119.2,128.1,129.3$ (2C), 129.7, 132.0 (2C), 132.1, 133.1, 134.6 (2C), 142.0 (2C), 150.9 (2C). HRMS Calcd for $\mathrm{C}_{19} \mathrm{H}_{11} \mathrm{~N}_{4} \mathrm{SCl}_{3}:[\mathrm{M}+\mathrm{Na}]^{+}, 454.9668$; Found: 454.9662 . 


\section{${ }^{13} \mathrm{C}$ NMR spectra of all compounds in Table 1 are given below}

RJ-43K-13C (SR)
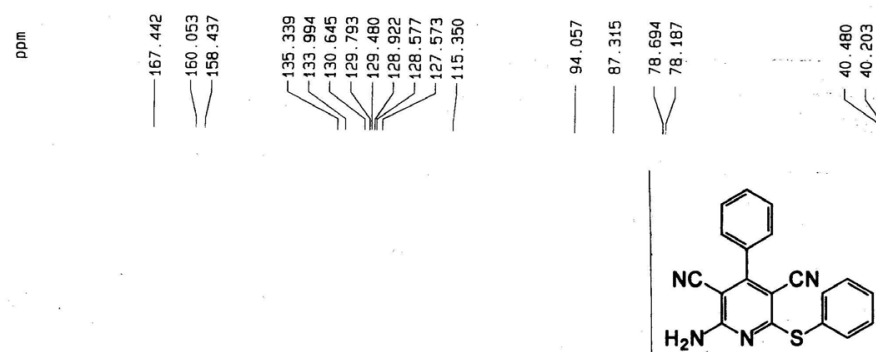

Entry 1, Table 1
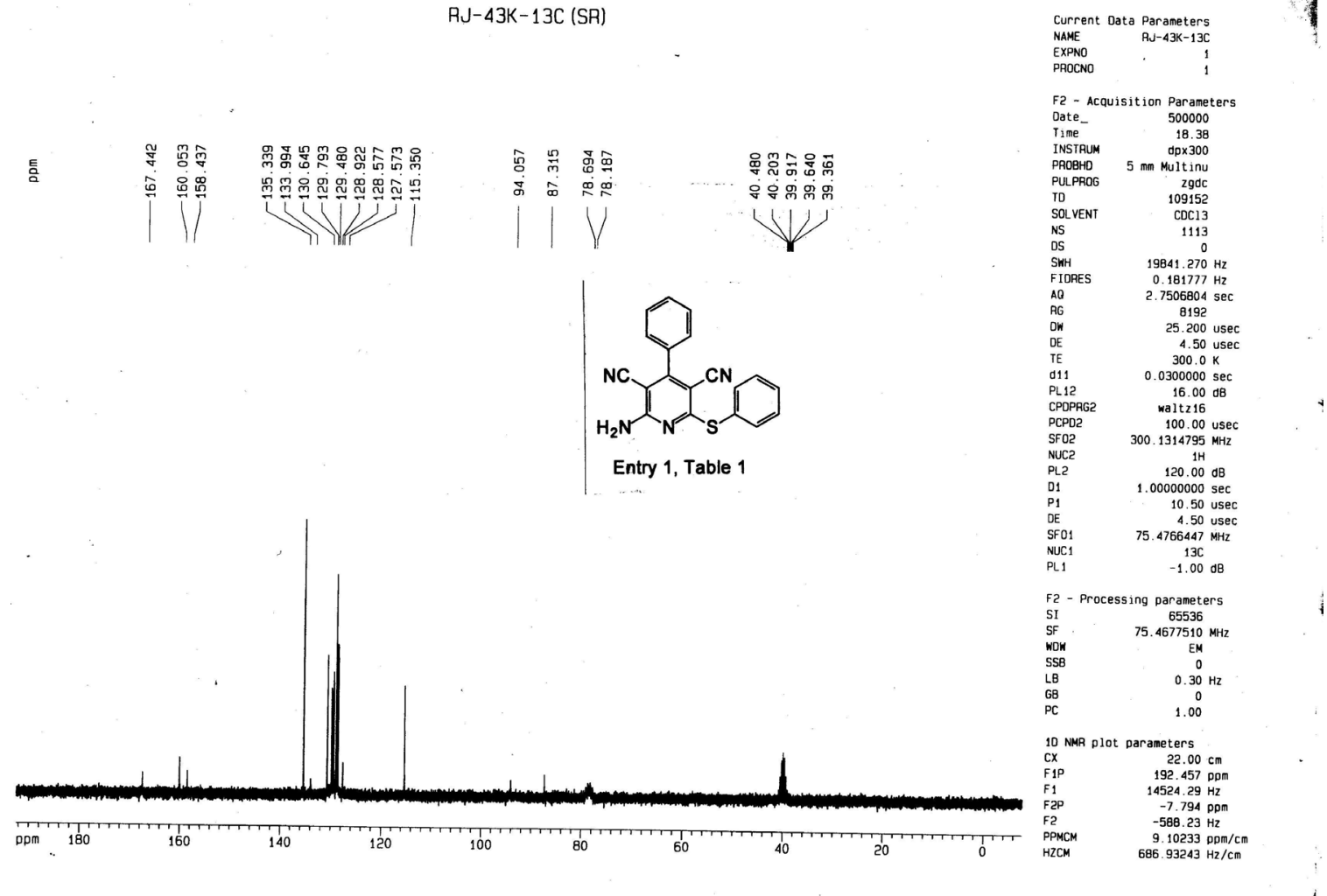

RJ-78K-13C (SR)
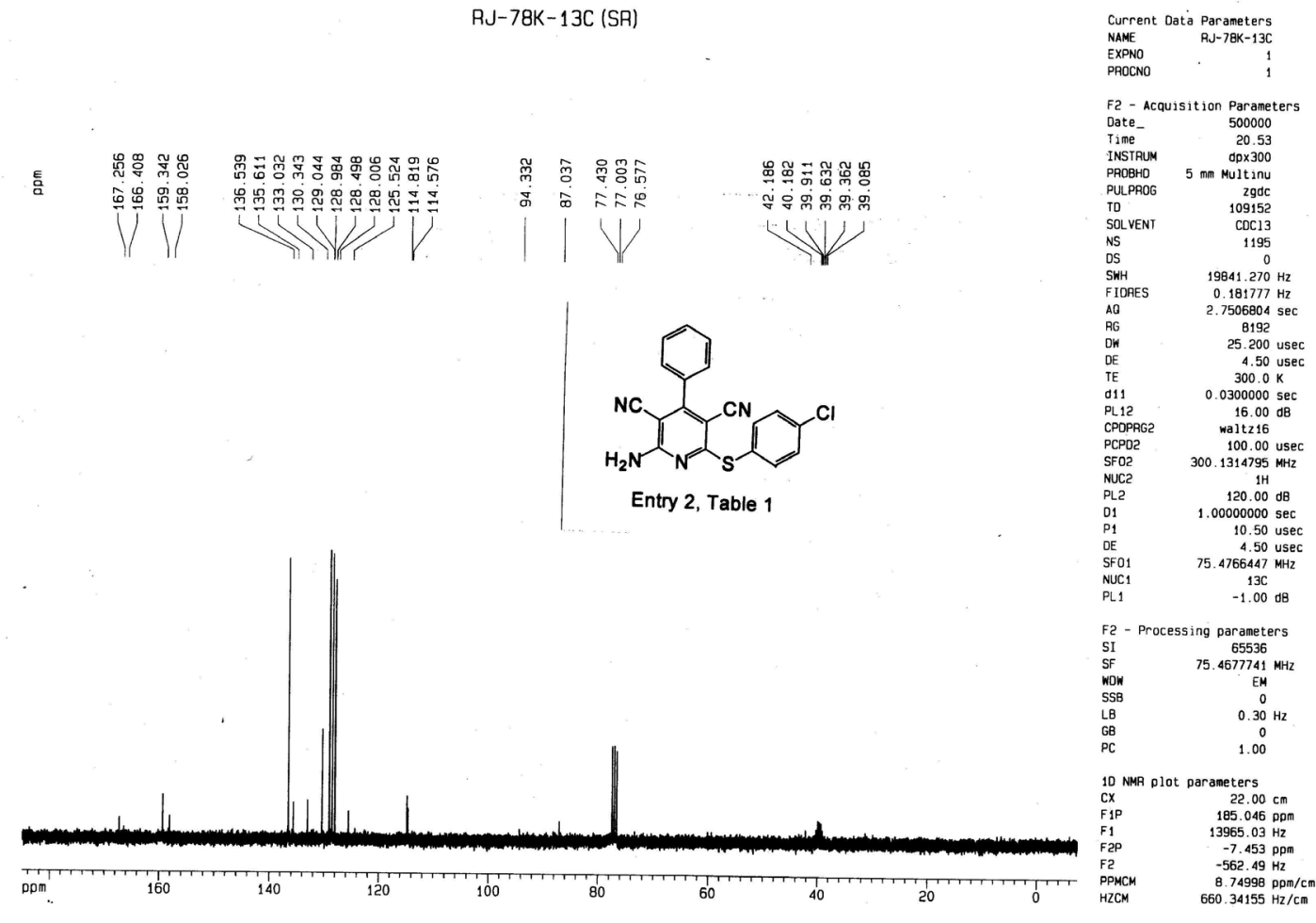


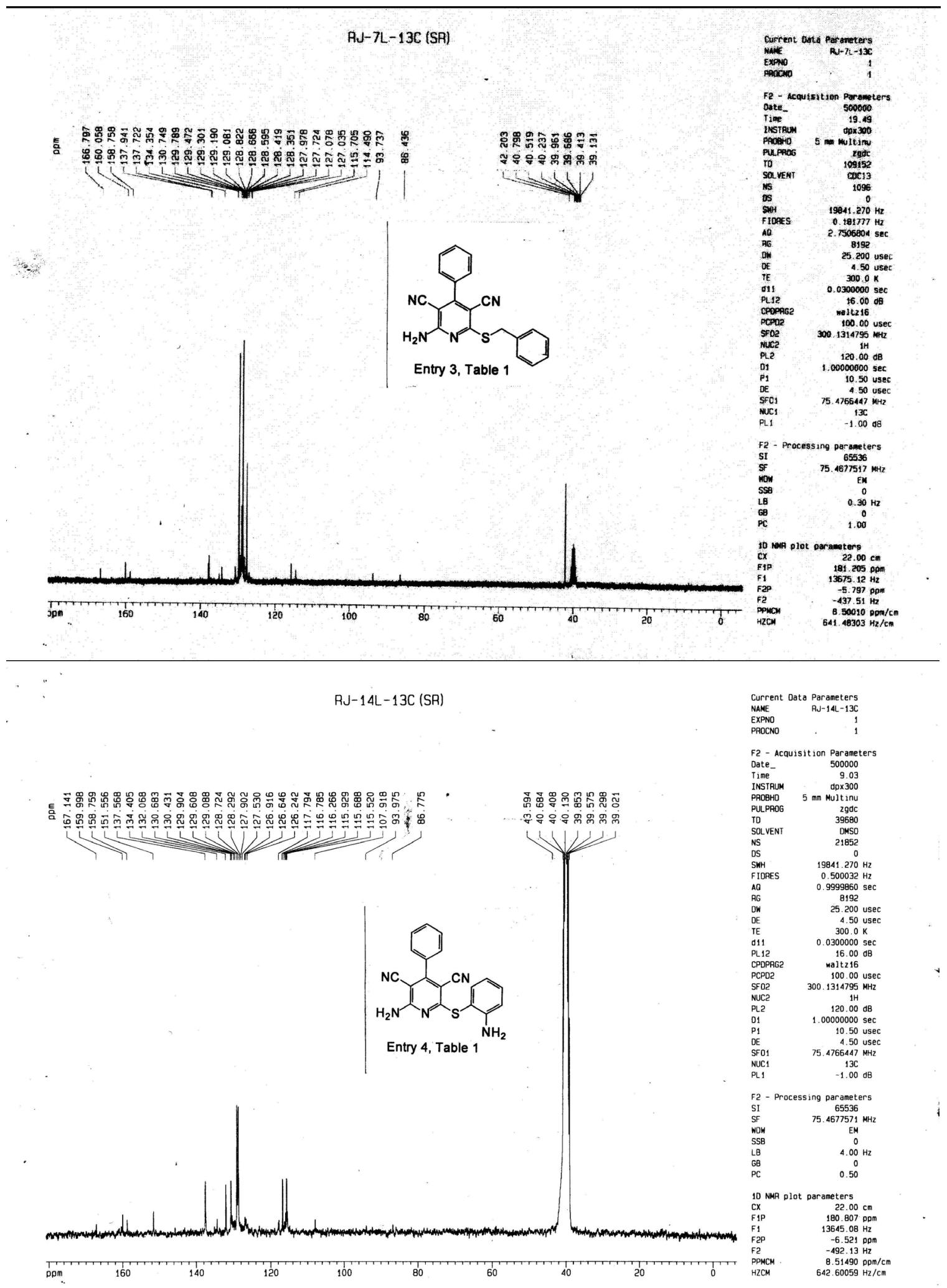


RJ-56K-13C (SR)

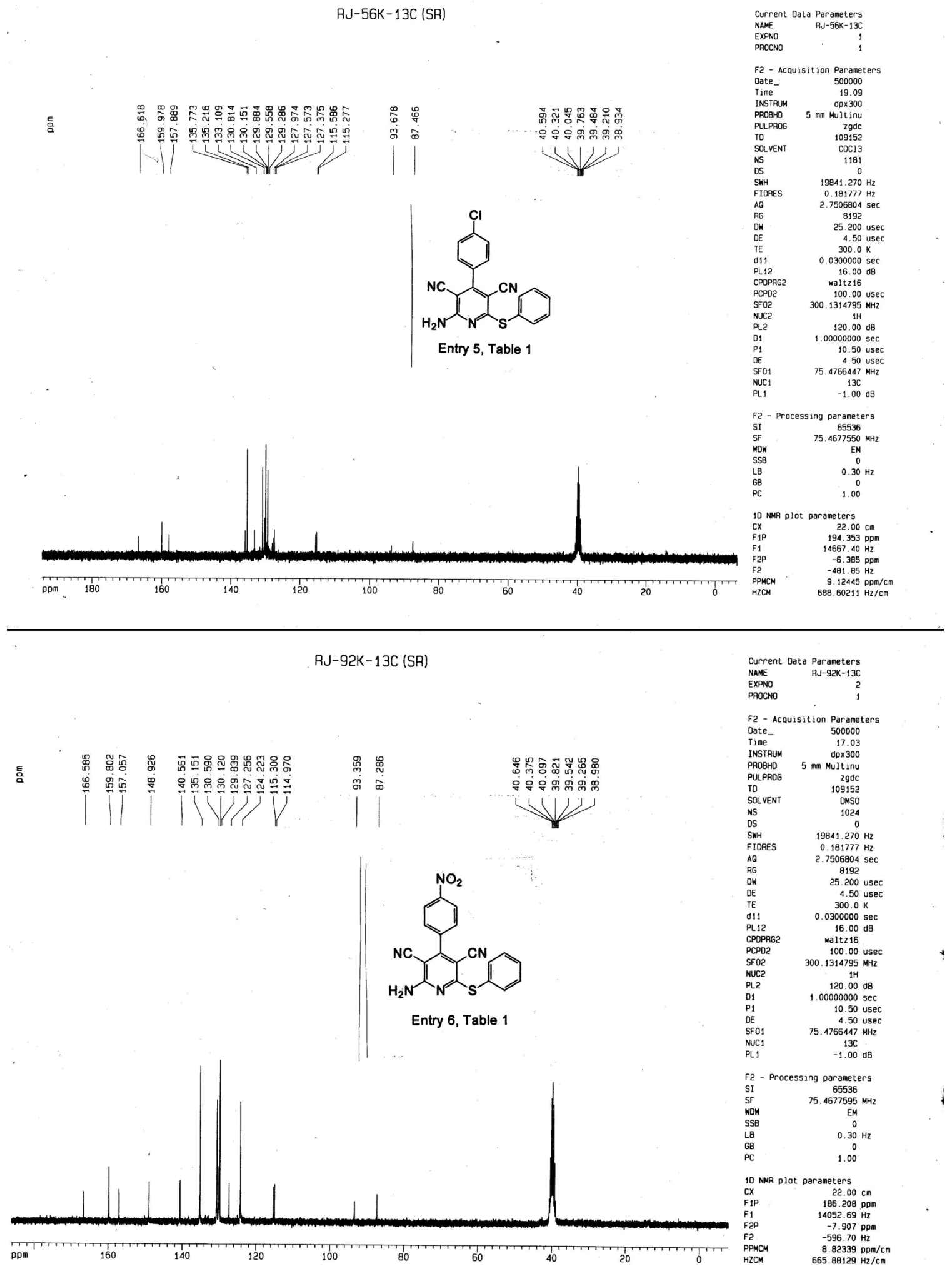




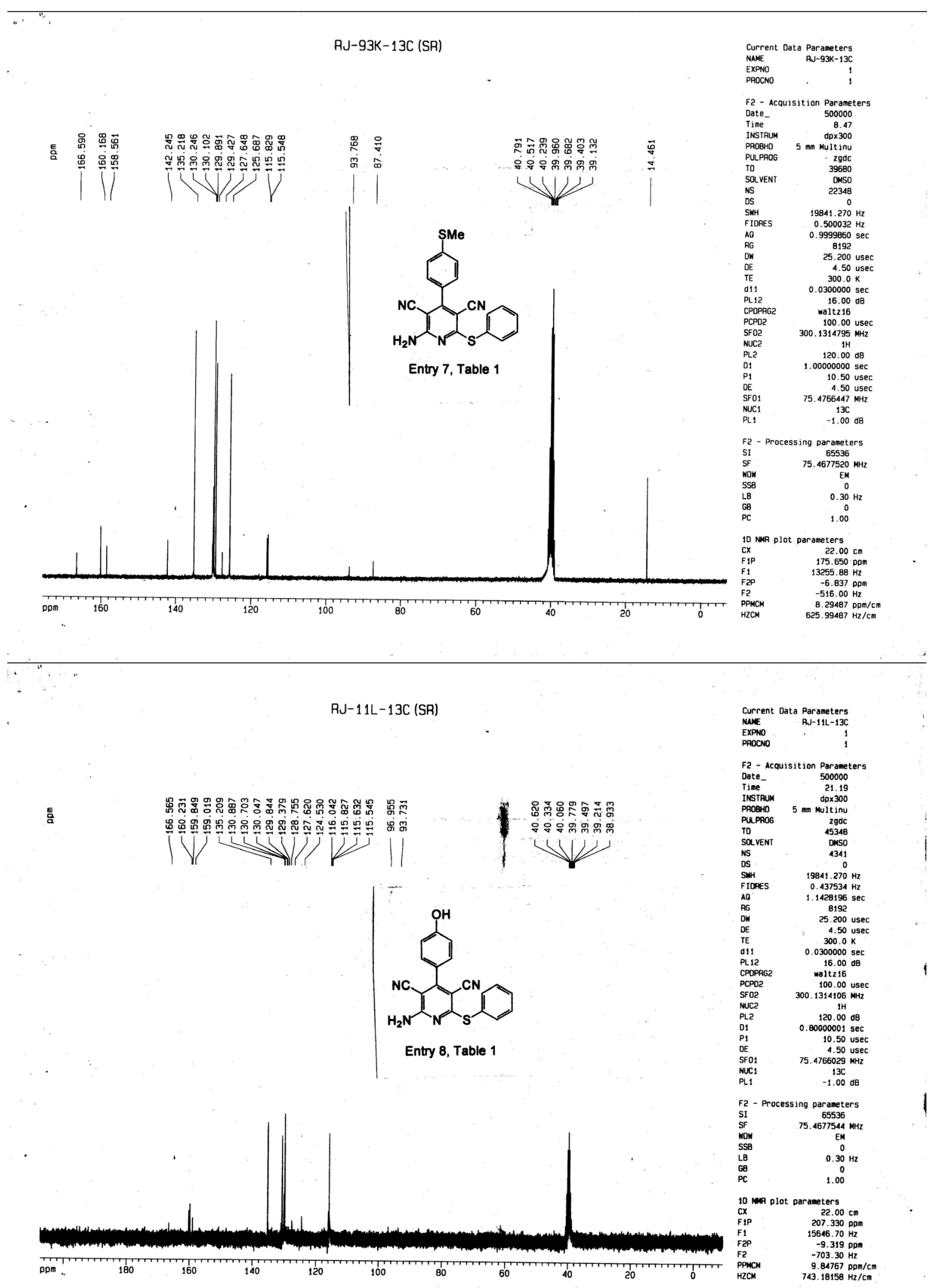


RJ91K-13C (SKD)

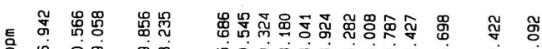

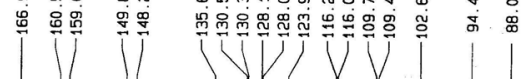
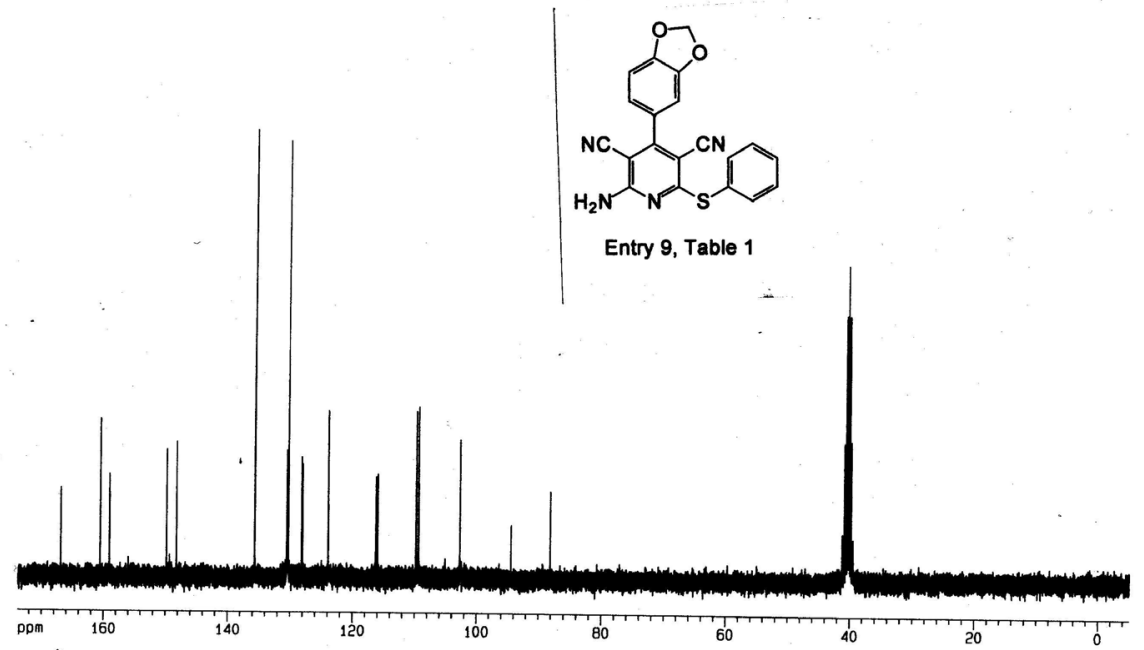

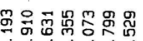

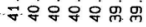

wy

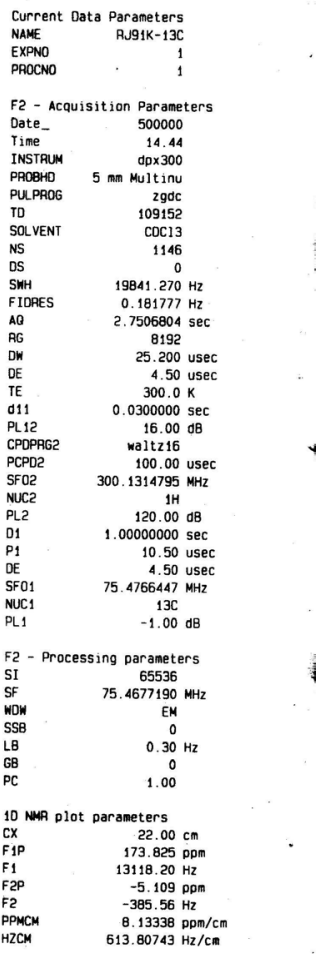

RJ $-49 K-13 C$ (SR)

E

Entry 10, Table 1

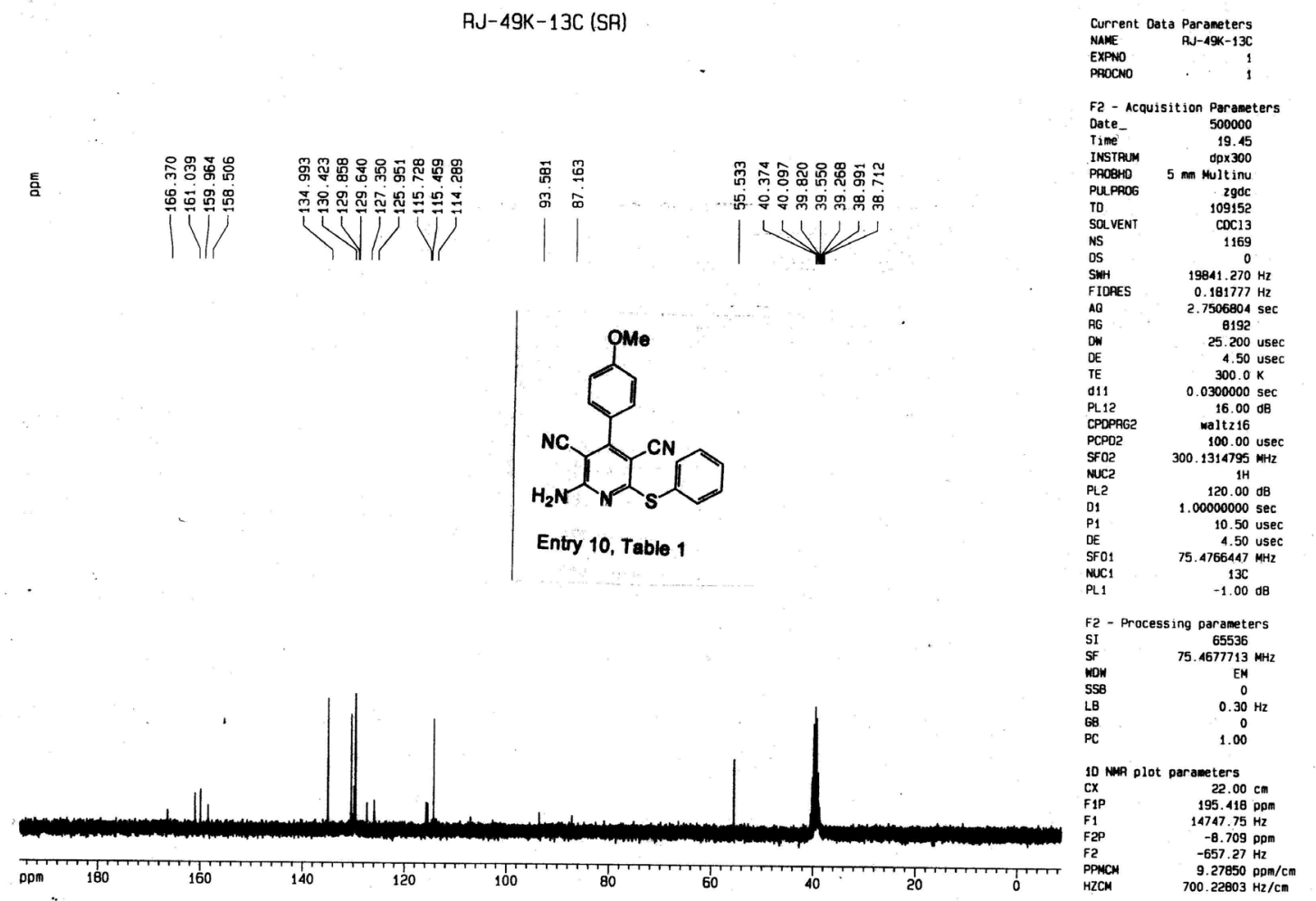




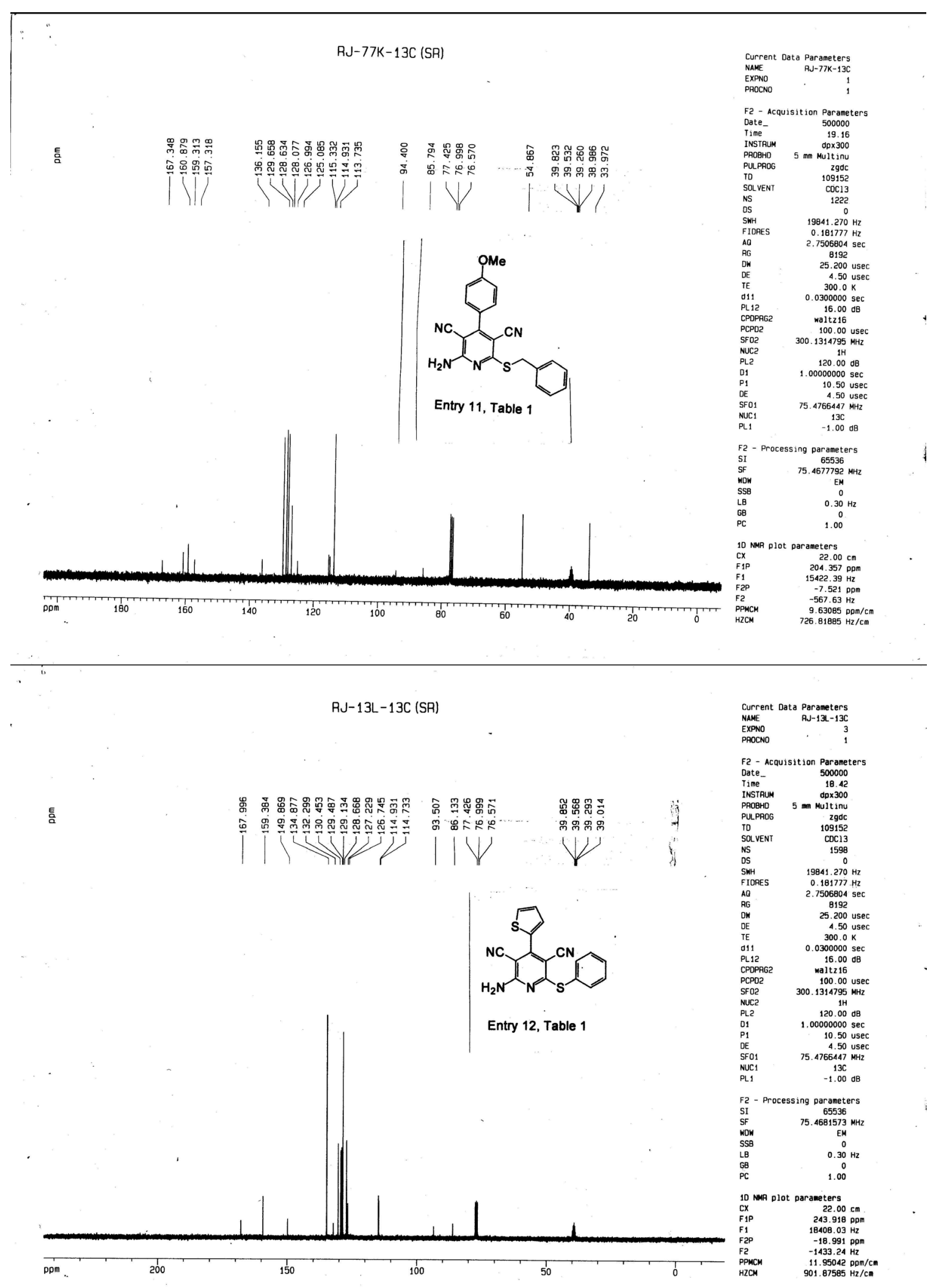




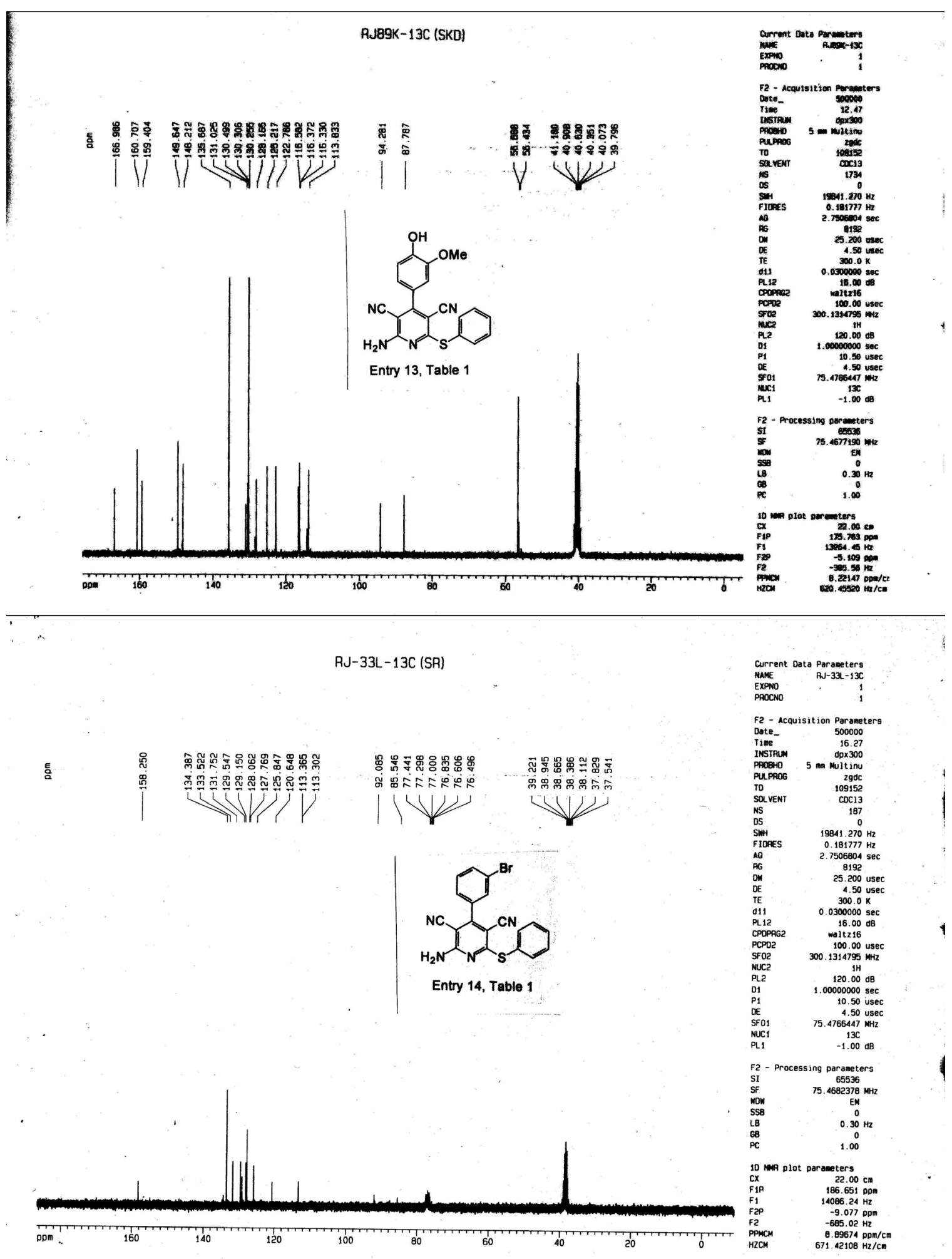


RJ $-34 L-13 C$ (SR)
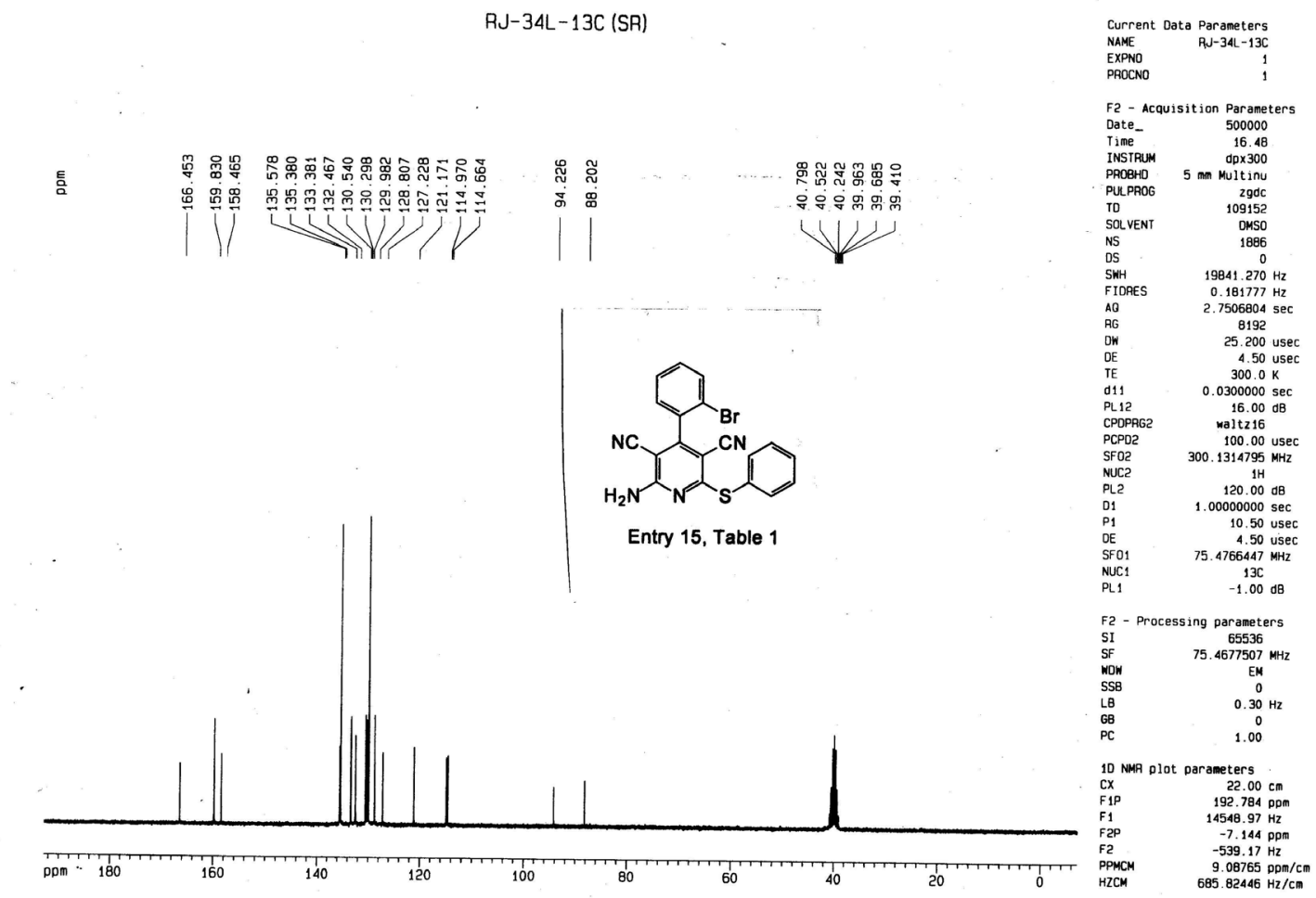

RJ110K-13C (SKD)

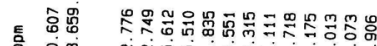

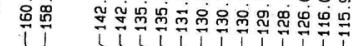

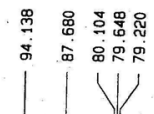

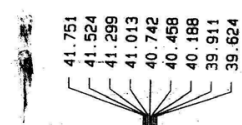

思

V
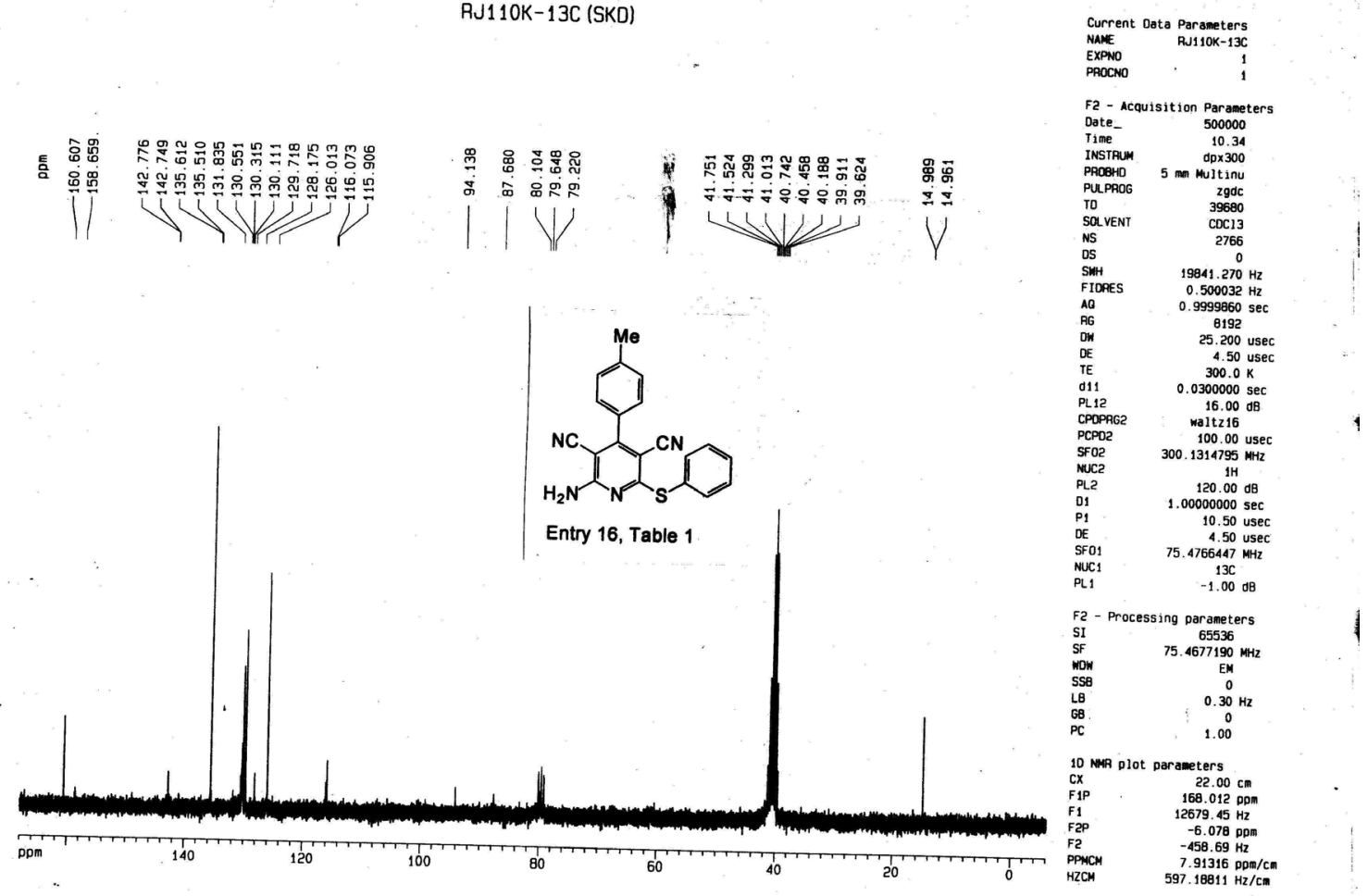

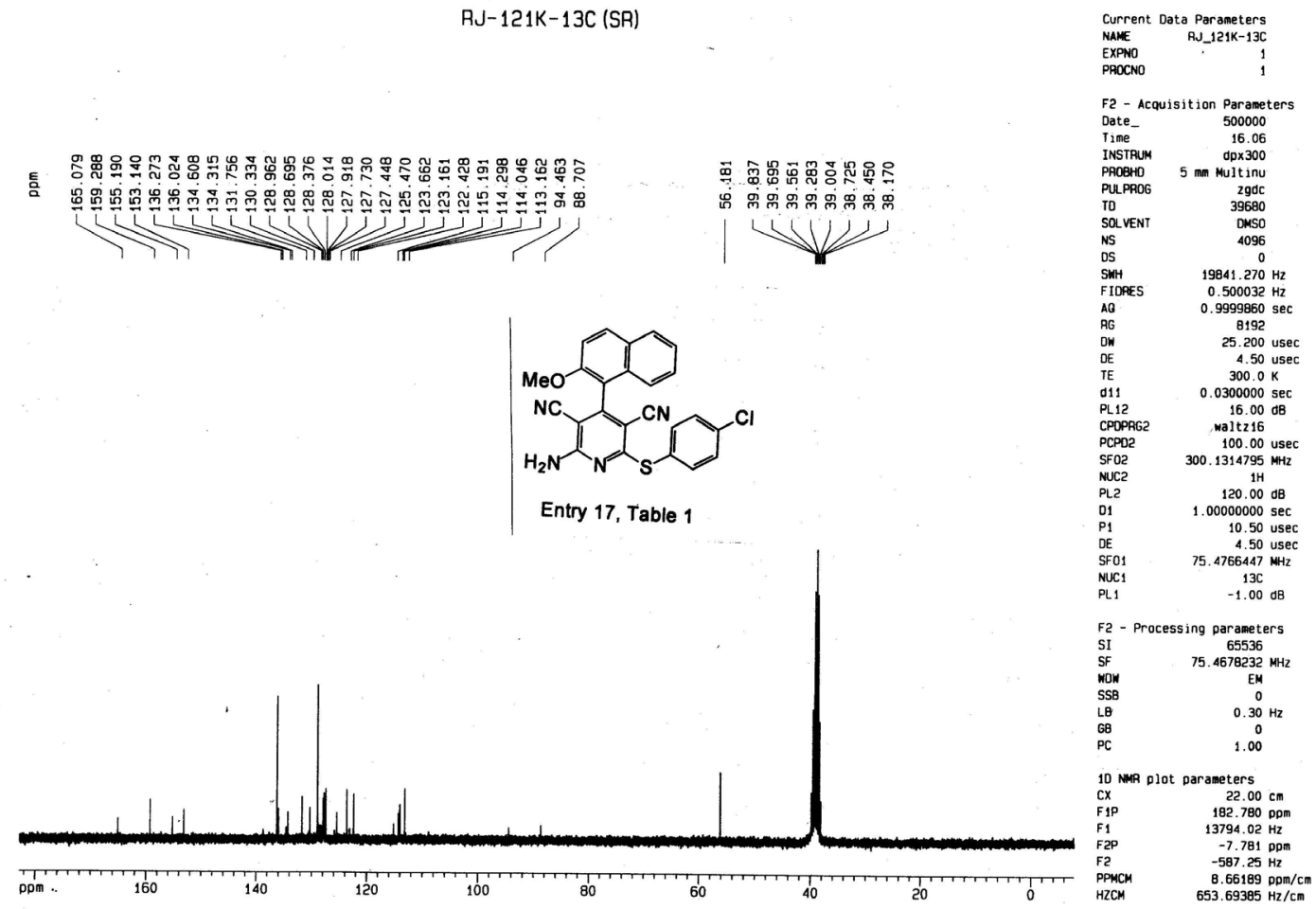

RJ136K-13C (SKD)
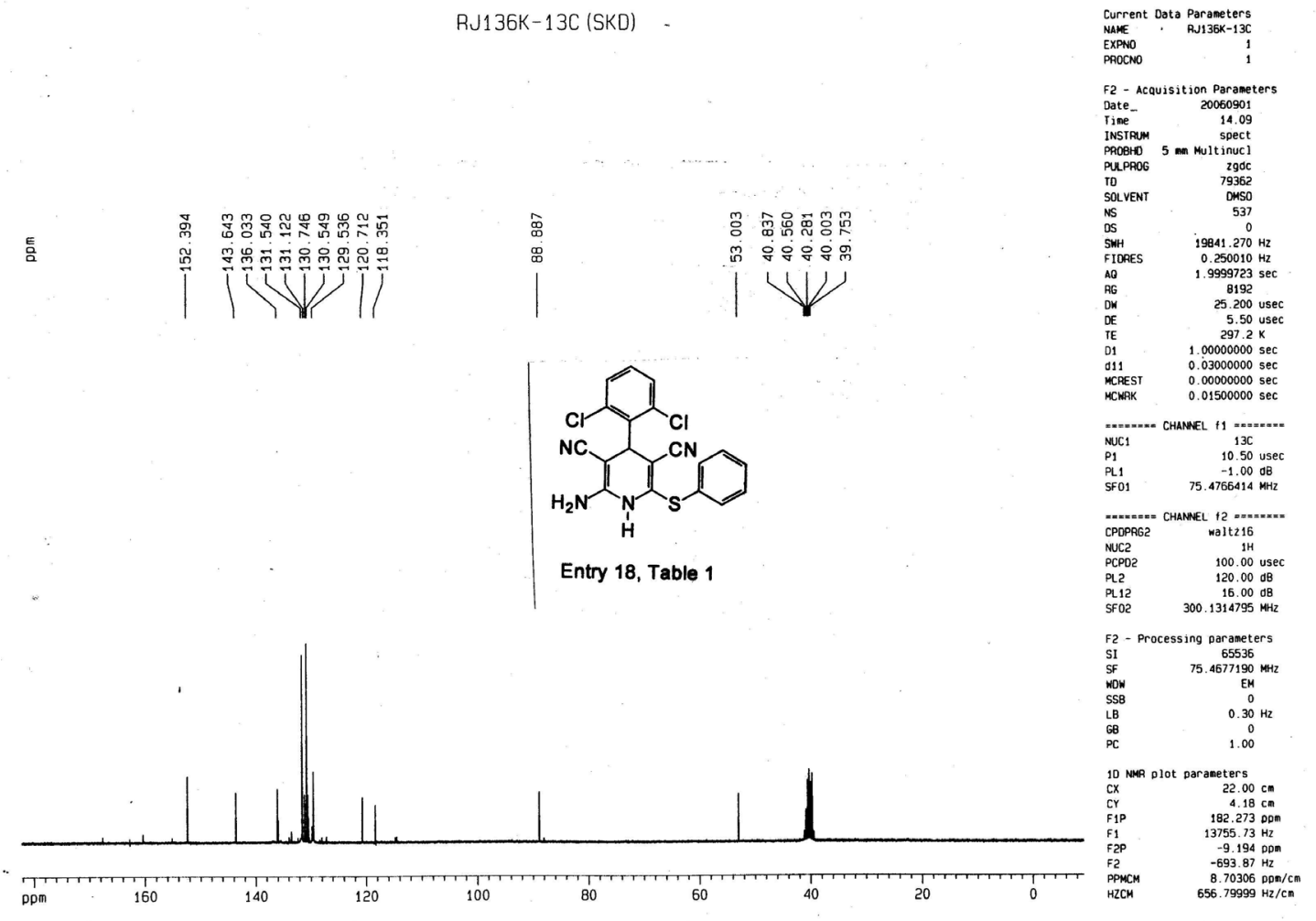
RJ-137K-13C (SR)

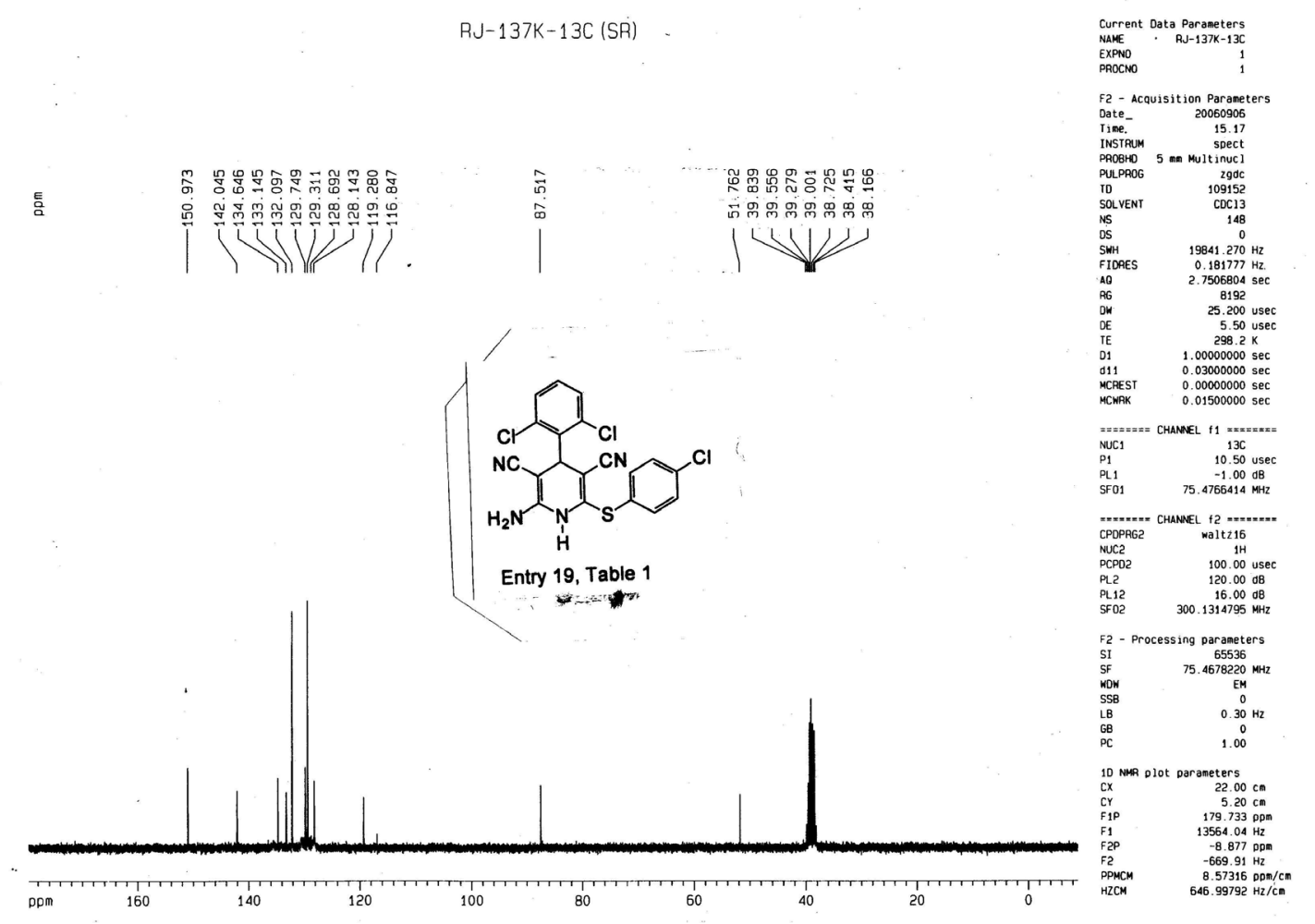

